\title{
Robot solutions for automated 3D surface measurement in production
}

\author{
Reinhard Danzl ${ }^{*}$, Thomas Lankmair ${ }^{1}$, Anne $\mathrm{Calvez}^{2}$, and Franz Helmi ${ }^{1}$ \\ ${ }^{1}$ Alicona, R\&D Department, 8074 Raaba/Graz, Austria \\ ${ }^{2}$ Alicona SARL, Gérante, 25000 Besançon, France
}

\begin{abstract}
With the increasing complexity of industrial parts, the requirements for $3 \mathrm{D}$ optical metrology are increasing in the production environment. Until recently, no high-resolution 3D optical metrology solution was available to measure surface roughness, surface defects and micro geometries on large and high precision parts. Until now, the only solutions for measuring such features on large parts were either portable tactile profilers for roughness measurement or the measurement of replicas. To simplify this type of measurement in production, a high-resolution $3 \mathrm{D}$ optical sensor has been mounted on a collaborative six-axis robot. The system allows an easy rough positioning with two-handle bars, fine positioning with integrated joysticks, fully automated measurement and subsequent measurement reporting including ok or not ok status. Typical applications include the measurement of the cutting edge geometry and quality on large tools such as milling cutters with shanks up to $1 \mathrm{~m}$ or the automated edge break measurement on large aerospace turbine discs up to $120 \mathrm{~kg}$. In addition to speeding up process series for the measurement of large parts, this solution also opens possibilities of closed loop manufacturing by integrating the robot sensor in an established machining solution. As an example a polishing or finishing cell that sends the measurement results to a machining solution and corrects the part if measurements are out of specification.
\end{abstract}

\section{Introduction}

The ongoing miniaturization of features on industrial parts and the reduction of manufacturing tolerances generate the need for highly accurate 3D measurement devices for measurements with sub- $\mu \mathrm{m}$ resolution. Whereas highly accurate measurements [1] have been made available for offline inspection in the laboratory in recent years, there is a strong focus towards automatic measurement in a production environment or inline to speed up the measurement time, to reduce user influence and to save costs. However, so far the combination of high resolution 3D sensors that can be automated and which are robust and fast enough for industrial environments are rarely found. Production-near measurement systems are typically only available for $2 \mathrm{D}$ inspection or for measurement with larger resolutions.

\footnotetext{
* Corresponding author: reinhard.danzl@alicona.com
} 
An additional challenge is that many parts are too large or too heavy to be measured with conventional metrology systems that have strong limits with respect to size and weight. Typically such large parts can be only measured by portable profilers for roughness measurement or alternatively by creating a replica of small portions of the large parts, which are then measured in the laboratory, a very time consuming process.

Here we present a novel metrology platform for industrial measurements that uses a unique combination of collaborative robots (cobots), a fast 3D sensor based on the FocusVariation principle [2] and software for automation of complex 3D measurements tasks. In contrast to conventional industrial robots, collaborative robots have the advantage that they can be easily moved by a human operator, so that no time-consuming and complex programming of measurement positions is necessary. Additionally, such robots immediately stop when they are exposed to an external force, such as a collision with a human operator, so that no severe injuries of human operators can happen.

After describing the basic 3D measurement technology based on the Focus-Variation principle we introduce two of four available hardware platforms, one for measuring large tools and one for measuring turbine. Afterwards we present results for one application in detail, the measurement of the geometry and quality of cutting edges on large milling cutters.

\section{3D Measurement of edges with collaborative robots}

\subsection{D Measurement using Focus-Variation}

The collaborative robots are equipped with a 3D sensor based on the Focus-Variation principle. The technique of Focus-Variation [2], [3] combines the small depth of focus of an optical system with vertical scanning to provide topographical and colour information from the variation of focus. Its vertical resolution depends on the chosen objective and can be as low as $10 \mathrm{~nm}$. The vertical scan range depends on the working distance of the objective and ranges from 10 to $34 \mathrm{~mm}$. The $\mathrm{x}-\mathrm{y}$ range is determined by the used objective and typically ranges from $0.4 \times 0.4 \mathrm{~mm}$ to $4 \times 4 \mathrm{~mm}$ for a single measurement. In addition to the scanned height data, Focus-Variation delivers a colour image with full depth of field which is registered to the $3 \mathrm{D}$ points. This provides an optical colour image which eases measurements as far as the identification and localization of measurement fields or distinctive surface features are concerned. The advantages of this sensor in terms of tool measurement are, that its vertical resolution down to $10 \mathrm{~nm}$ and its ability to measure also steep surface flanks makes it suitable for measuring the geometry and the roughness of the cutting edges of the inspected tools.

\subsection{Collaborative robot for measuring large tools}

One available hardware platform is designed for the measurement of large tools, such as drills, milling cutters etc. with shank lengths up to $1 \mathrm{~m}$ (Fig. 1.). The system consists of a collaborative robot, the $3 \mathrm{D}$ sensor based on the Focus-Variation technology, a motorized zstage and a rotation table that is equipped with a standardized taper interface.

The whole system is placed on wheels so that the tool to be measured can be either clamped in the rotation unit, or that the system can be moved to the manufacturing machine for inline measurement. 
On the sensor a smartphone is mounted, which includes an app showing the live view of the sensor, so that the robot can be easily focused on the surface without the need to look at the large monitor that is also available.

One of the key applications to be realised on this platform is the inspection of the quality and geometry of the cutting edges [4] of large tools. In contrast to traditional measurement devices in the laboratory, where each cutting insert of the tool has to be removed from the milling cutter, then placed below the laboratory measurement device, measured and reinserted again, the process cycle time can be dramatically reduced with the new system. The whole tool can be measured as it is, or even within the manufacturing machine.

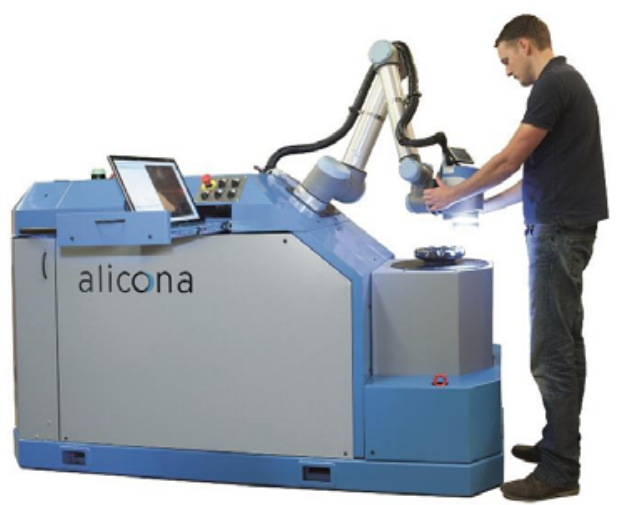

Fig. 1. A hardware platform designed for measuring large tools with shanks up to 1 meter, consisting of a collaborative robot, equipped with a high-resolution 3D sensor. Operating and programming is easy and intuitive.

\subsection{Collaborative robot for measuring turbine discs}

Another hardware platform is designed for the quality assurance of turbine discs. In addition to the collaborative robot and the $3 \mathrm{D}$ sensor, the system consists of a rotation unit able to carry parts up to $120 \mathrm{~kg}$. This system is also equipped with a sensor that stops the machine when a user enters the measurement area.

One key application here is to evaluate the edge break on large aerospace turbine discs to assure that none of the edges are too sharp to compromise aircraft safety.

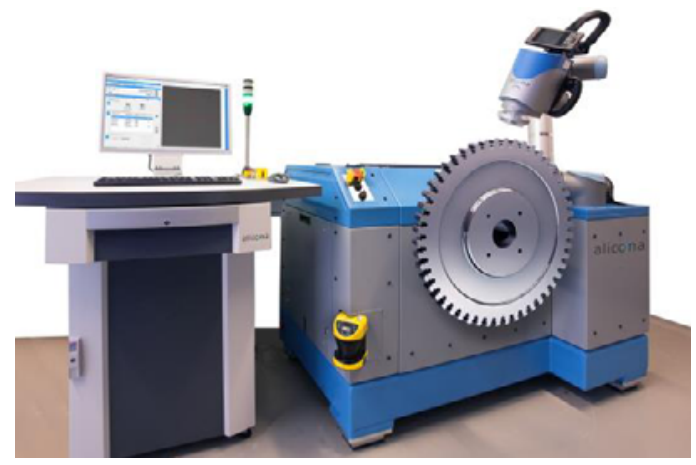

Fig. 2. A hardware platform designed for measuring turbine discs with weight up to $120 \mathrm{~kg}$, consisting of a collaborative robot, equipped with a high-resolution 3D sensor. The robot arm, with its attached measuring sensor, is easily and conveniently manipulated by the operator. 


\subsection{Automatic measurement of the quality of cutting edges}

A key application for the proposed hardware platform in subsection 2.2 is the measurement of cutting edges on large tools such as milling cutters. Besides the measurement of the edge geometry (edge radius, wedge angle, ...), tool manufacturers are specially interested if the cutting edge has defects either before use in the manufacturing machine or during their lifetime. In this context, the proposed measurement device offers the possibility to measure a large range of defect parameters such as the length, area or volume of edge defects. The system is equipped with a software interface that allows automating the whole measurement process. Hereby, the administrator once defines a measurement program that specifies at which positions the different edges must be measured and which specific parameters should be inspected. Afterwards the operator performs the measurements using the following steps:

1. The operator clamps the whole milling cutter that is equipped with a series of cutting inserts in the rotation table.

2. The operator selects the measurement program and starts it.

3. The measurement is performed automatically and the results are displayed on the screen.

The process of measuring the parameters which quantify if the edge has defects or not is performed using the following steps:

1. Performing a 3D measurement of the cutting edge using the optical 3D sensor.

2. Either calculating a reference geometry or using a reference geometry (e.g. from CAD or from a golden standard) as comparison.

3. Calculating the difference between the 3D measurement and the reference geometry.

4. Calculating a range of parameters that quantify the quality of the cutting edges.

One of the key aspects is that for edges with small defects, it is not necessary to quantify the defects by comparing them to a reference edge (step 2 above), but the system can automatically estimate the reference geometry based on the measured data in the case that $50 \%$ of the measured edge are free from defects. This is visualized in Fig. 3. which shows the measured 3D dataset of a cutting edge and the automatically calculated reference geometry.

Once the difference between the measured dataset and the reference dataset has been obtained, a series of parameters can be evaluated including defect length, area and volume. Some of these are graphically visualized in Fig. 4.
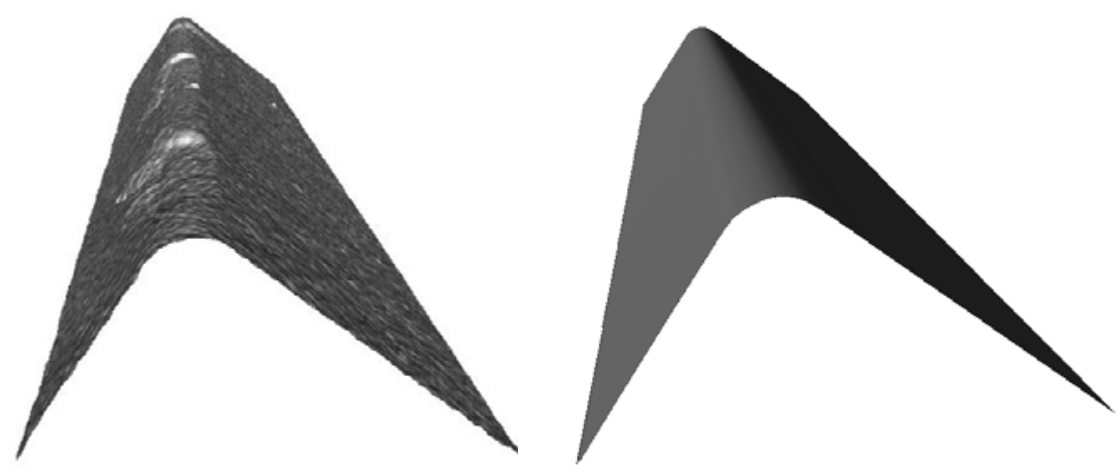

Fig. 3. Measured 3D dataset (left) and calculated reference geometry (right). 


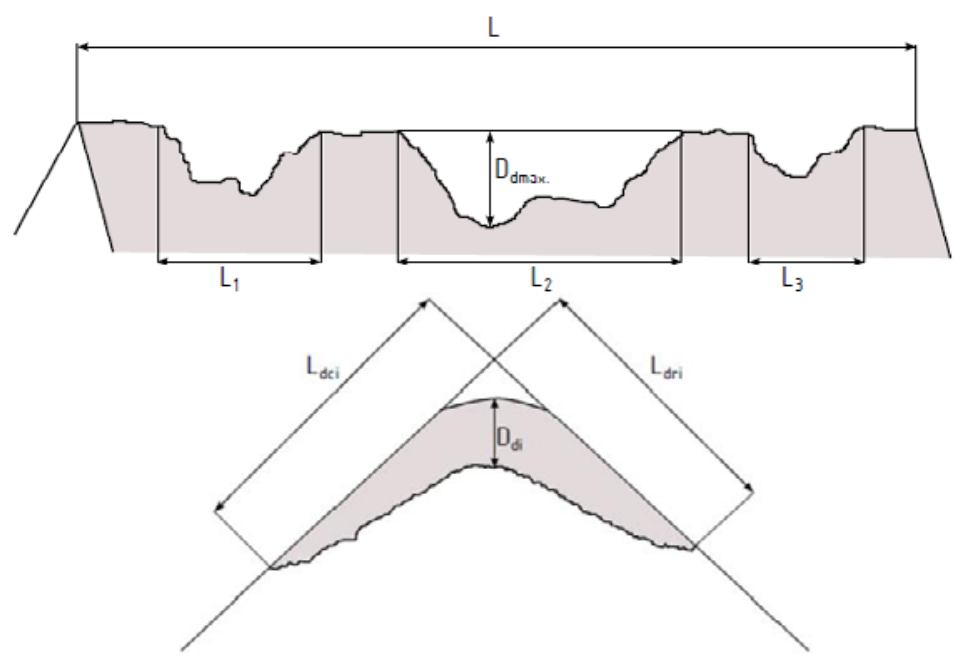

Fig. 4. An overview of several possible parameters for quantifying the quality of a cutting edge. Top: parameters that quantify the chipping along the cutting edge. Bottom: Cross-section of the cutting edge showing parameters that describe the defect length along the clearance and chipping surface.

\section{Results}

In the following we present results of quality measurements of cutting edges which have been realized using the cobot solution in Fig. 1. Fig. 5. shows the 3D dataset of a measured cutting edge with overlaid true colour information (left) where found defects have been marked with a bright surrounding. The figure on the right-hand side, shows the depth of the defects with pseudo colours where the deepest defect is about $5 \mu \mathrm{m}$. In total, five defects have been found, whereas the threshold for the defects has been set to $1 \mu \mathrm{m}$.

In Fig. 6. a height profile along the cutting edge is provided. In addition to the defects, also the geometry of the cutting edge including the cutting edge radius or wedge angle is evaluated as shown in Fig. 7. To verify that the automatically detected parameters are correct, the parameters have also been manually measured, e.g. using cross-sections and manual measurements as shown in Fig. 8., where the defect length along the chipping and clearance surface has been measured. The difference of the automated and manual measurement for different parameters, as well as the repeatability of 10 automated measurements was smaller than $1 \mu \mathrm{m}$.
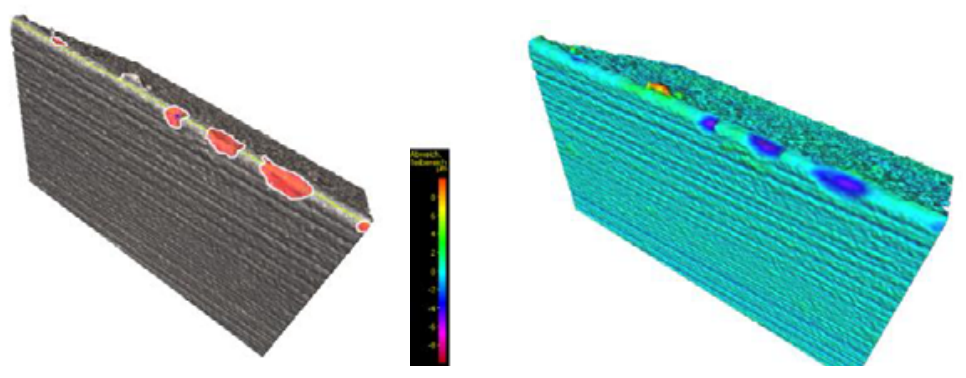

Fig. 5. Measured 3D dataset with true colour information and marked defects (left) and with pseudo colours, which show the difference to the reference geometry (right). 


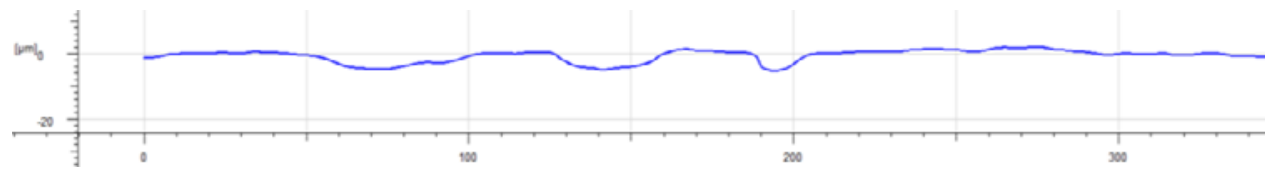

Fig. 6. Height profile which has been extracted along the cutting edge.

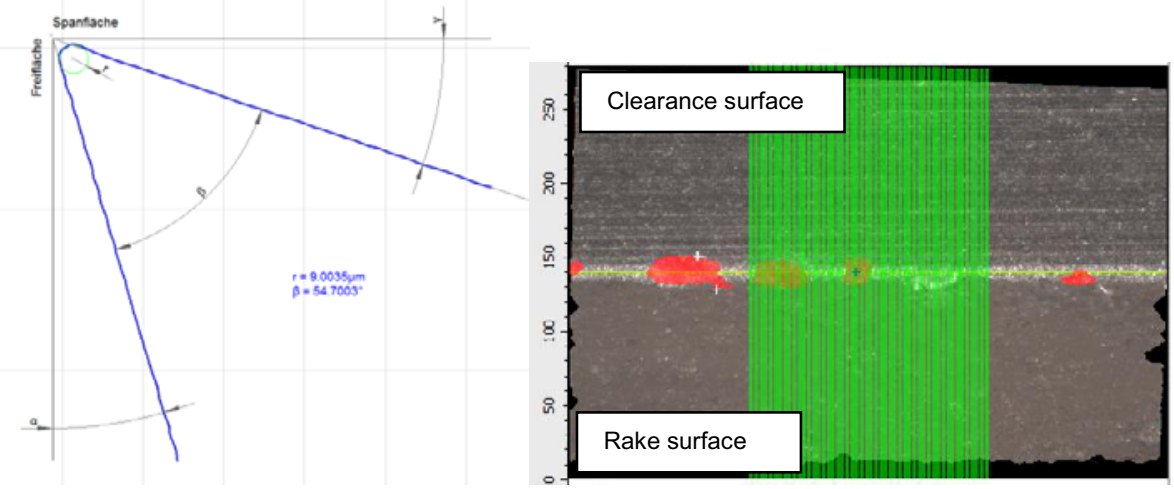

Fig. 7. Mean cutting edge profile (left) and profile paths for profile extraction (right).

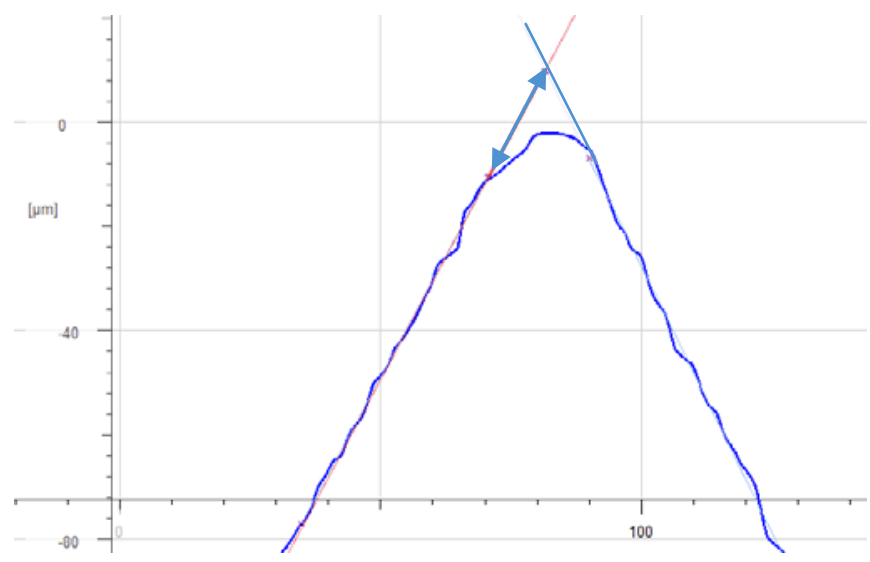

Fig. 8. Left: Manually evaluated defect length along the clearance surface.

\section{Conclusions}

We have proposed new measurement systems for the measurement of large high precision parts within a production environment. The systems equipped with collaborative robots and high resolution $3 \mathrm{D}$ sensors are designed for easy and automated measurements that can be also performed by non-expert users. A key application, the measurement of the cutting edge quality on large tools, is realised with high accuracy and repeatability. In contrast to traditional solutions, the process measurement cycle time can be dramatically reduced, since tedious unmounting of cutting inserts and individual measurement on a conventional laboratory measurement device is exchanged by fully automated measurement of all cutting edges in a row without any user interaction. 


\section{References}

1. R.K. Leach, Springer, Optical Measurement of Surface Topography 1 (2011)

2. ISO/CD 25178-606, Geometrical product specification (GPS) - Surface texture: Areal Part 606: Nominal characteristics of non-contact (focus variation) instruments (2015)

3. R. Danzl, F. Helmli, S. Scherer, Journal of Mech. Eng., Focus variation - A robust technology for high resolution optical 3D surface metrology 57(3), 245-256 (2011)

4. M. Prantl, R. Danzl, F. Helmli, Kassel University Press, Optische 3D Messung der Schneidkantenverrundung, Schneidkantenpräparation, Ziele Verfahren und Methoden, 163-179 (2009) 